\title{
ON CRITERIA FOR UNIQUE EXTREMALITY OF TEICHMÜLLER MAPPINGS
}

\author{
EDGAR REICH ${ }^{1}$
}

\section{Introduction}

Let $\varphi_{0}(z)$ be a complex-valued measurable function defined in a region $\Omega$ of the complex plane $C, \varphi_{0}(z) \neq 0$ a.e., and let $k$ be a constant, $0<k<1$. A quasiconformal mapping $w=f(z)$, of $\Omega$, will be said to be of Teichmüller type ${ }^{2}$ if $f$ has complex dilatation

$$
\varkappa(z)=\frac{f_{\bar{z}}}{f_{z}}=k \frac{\overline{\varphi_{0}(z)}}{\left|\varphi_{0}(z)\right|}, \quad z \in \Omega .
$$

We are interested in whether $f$ is uniquely extremal among the collection of quasiconformal mappings of $\Omega$ that are in the same homotopy class as $f$, and whose pointwise boundary values are the same as those of $f$. Let $\mathscr{B}(\Omega)$ be the Banach space consisting of holomorphic functions $\varphi(z)$ belonging to $\mathscr{L}^{1}(\Omega)$, with norm

$$
\|\varphi\|=\iint_{\Omega}|\varphi(z)| d x d y<\infty, \quad \varphi \in \mathscr{B}(\Omega) .
$$

For $\varphi \in \mathscr{B}(\Omega)$, set

$$
\delta\{\varphi\}=k\|\varphi\|-\operatorname{Re} \iint_{\Omega} x(z) \varphi(z) d x d y .
$$

We recall [5]

The orem A. Suppose there exists a sequence of functions $\varphi_{n} \in \mathscr{B}(\Omega), n=1,2, \ldots$, such that (1.2) and (1.3) hold; namely,

$$
\begin{gathered}
\lim _{n \rightarrow \infty} \varphi_{n}(z)=\varphi_{0}(z) \quad \text { pointwise a.e. in } \Omega, \\
\lim _{n \rightarrow \infty} \delta\left\{\varphi_{n}\right\}=0 .
\end{gathered}
$$

Then $f$ is uniquely extremal.

1 Work done with support from National Science Foundation Grant MCS-7728149, Amendment No. 02.

${ }^{2}$ We deviate from the usual terminology in not a-priori requiring $\varphi_{0}$ to be holomorphic in $\Omega$. Whether or not non-holomorphic $\varphi_{0}$ exist for which the hypotheses of Theorems A or B hold is an open question. 
While Theorem A enjoys properties of simplicity and rather wide usefulness, it is not known whether it represents a necessary and sufficient condition for unique extremality of Teichmüller-type mappings. In particular, in a certain borderline case [5, Section 2, $\alpha=3$ ] of mappings covered by Theorem A which is known on other grounds to be uniquely extremal, it is unclear whether or not Theorem A applies. Our current contributions will include showing that (1.3) can be replaced by the weaker assumption of boundedness of $\left\{\delta\left\{\varphi_{n}\right\}\right\}$, provided (1.2) is strengthened appropriately. This is done in Theorem B which is stated below. From Sections 4 and 5 it will be seen that Theorem B succeeds in allowing conclusions regarding unique extremality for classes of mappings like the case referred to above where the applicability of Theorem A is doubtful.

Theorem B. Suppose there exists a sequence of functions $\varphi_{n} \in \mathscr{B}(\Omega), n=1,2, \ldots$, such that (1.4), (1.5), and (1.6) hold; namely,

$$
\begin{gathered}
\lim _{n \rightarrow \infty} \varphi_{n}(z)=\varphi_{0}(z) \quad \text { pointwise a.e. in } \Omega, \quad \varphi_{0} \in \mathscr{L}_{\mathrm{loc}}^{1}(\Omega), \\
\delta\left\{\varphi_{n}\right\} \leqq M, \quad n=1,2, \ldots,
\end{gathered}
$$

$(1.6)^{3} \varliminf_{A \rightarrow \infty} \iint_{\Omega(n, A)}\left|\varphi_{n}(z)\right| d x d y=0, \quad$ uniformly with respect to $n$ where

$$
\Omega(n, A)=\left\{z \in \Omega:\left|\varphi_{n}(z)\right|>A\left|\varphi_{0}(z)\right|\right\} .
$$

Then $f$ is uniquely extremal.

The computations required for the proofs are elaborate, but it has been possible to clarify the methods by effectively separating the parts of the proof in which the analyticity of the $\varphi_{n}$ 's play a role from more formal $\mathscr{L}^{1}$ aspects. We will reprove Theorem A in the process (Section 3 ) of proving Theorem B. In fact, all results of [5] are subsumed in results obtained in the present paper.

After the examples in Sections 4 and 5, we turn, in Section 6 to the linear functional, $\Lambda_{\varkappa}$,

$$
\Lambda_{\varkappa}[\varphi]=\iint_{\Omega} x(z) \varphi(z) x d y, \quad \varphi \in \mathscr{B}(\Omega) .
$$

Recall the fact [5, Theorem 3] that the hypothesis of Theorem A also implies that $\Lambda_{x}$ has a unique Hahn-Banach extension from $\mathscr{B}(\Omega)$ to $\mathscr{L}^{1}(\Omega)$. It turns out that this is no accident. By systematizing the approach we are able to conclude (Theorem $C$, Section 6) that any of a large class of sufficient conditions for unique extremality of quasiconformal mappings is also sufficient for the unique HahnBanach extension of $\Lambda_{\varkappa}$. (However, the problem as to whether uniqueness of the Hahn-Banach extension of $\Lambda_{x}$, when $\left\|\Lambda_{x}\right\|=\|x\|_{\infty}$, is necessary and sufficient for the unique extremality of $f$ is still open.)

\footnotetext{
${ }^{3}$ If for some $A>0,\left|\varphi_{n}(z)\right| \leqq A\left|\varphi_{0}(z)\right|, n=1,2, \ldots, z \in \Omega$, then the requirement (1.6) is vacuous.
} 


\section{Versions and consequences of the main inequality}

Let $w=f(z)$ be any quasiconformal mapping of $\Omega$ (not necessarily of Teichmüller type), and let $\chi(z)=f_{\bar{z}} / f_{z}$ be its complex dilatation. Let $\hat{x}(w)$ denote the complex dilatation of $f^{-1}(w)$. Let $w=g(z)$ be a quasiconformal mapping of $\Omega$ with the same pointwise boundary values as $f(z)$, and in the same homotopy class; let $x_{1}(w)$ be the complex dilatation of $g^{-1}(w)$. If $\hat{x}(w)$ and $x_{1}(w)$ are evaluated at $w=f(z)$, then it is known $[2,6]$ that the "Main Inequality"

$$
\begin{aligned}
& \iint_{\Omega}|\varphi(z)| d x d y \leqq \iint_{\Omega}|\varphi(z)| \frac{\left|1-\varkappa(z) \frac{\varphi(z)}{|\varphi(z)|}\right|^{2}}{1-|\chi(z)|^{2}} . \\
& \frac{\mid 1+\varkappa(z) \frac{\varkappa_{1}(w)}{\hat{\chi}(w)} \cdot \frac{\varphi(z)}{|\varphi(z)|} \cdot\left[\frac{1-\overline{\chi(z)} \frac{\overline{\varphi(z)}}{|\varphi(z)|}}{1-\varkappa(z) \frac{\varphi(z)}{|\varphi(z)|}}\right]}{1-\left|\varkappa_{1}(w)\right|^{2}} d x d y,
\end{aligned}
$$

holds for all $\varphi \in \mathscr{B}(\Omega)$. We introduce

$$
\alpha(z)=\hat{\varkappa}(f(z)), \quad \mu(z)=\varkappa_{1}(f(z)) .
$$

Evidently, in view of the relation between $f$ and $f^{-1}$,

$$
|\alpha(z)|=|\varkappa(z)| \text { a.e. in } \Omega .
$$

Suppose the notation (2.1) is introduced in $\left(\mathrm{M}^{*}\right)$, and (2.2) is assumed to hold. We shall then refer to the resulting formal inequality involving $\Omega$, and the functions $\varphi, \alpha, x, \mu$, where we suppose that $\|\varkappa\|_{\infty}<1,\|\mu\|_{\infty}<1, \varphi \in \mathscr{L}^{1}(\Omega)$, as the inequality (M). When referring to this formal inequality we do not necessarily require $\varphi$ to belong to $\mathscr{B}(\Omega)$, nor do we interpret the $\mathscr{L}^{\infty}$ functions $x(z), \alpha(z), \mu(z)$, in any manner relating to the mappings $f$ and $g$, beyond requiring that (2.2) holds.

We will now proceed to rewrite (M) in several ways. The integral on the right side of $(\mathrm{M})$ is of the form

where

$$
\iint_{\Omega} \lambda|\varphi| \frac{d x d y}{\left(1-|\varkappa|^{2}\right)\left(1-|\mu|^{2}\right)}
$$

$$
\lambda=\left|1-\varkappa \frac{\varphi}{|\varphi|}+\frac{\varkappa \mu}{\alpha} \frac{\varphi}{|\varphi|}\left(1-\bar{\varkappa} \frac{\bar{\varphi}}{|\varphi|}\right)\right|^{2}=\left|(1-\bar{\alpha} \mu)-\left(\frac{\alpha-\mu}{\alpha}\right) \varkappa \frac{\varphi}{|\varphi|}\right|^{2} .
$$

After some simplification, and use of (2.2), one finds that

$$
\lambda-\left(1-|x|^{2}\right)\left(1-|\mu|^{2}\right)=2|\alpha-\mu|^{2}-2 \operatorname{Re}\left[\left(\frac{\varkappa}{\alpha}-\bar{\mu} x\right)(\alpha-\mu) \frac{\varphi}{|\varphi|}\right] .
$$


Thus, (M) is equivalent to the inequality

$\left(\mathrm{M}^{\prime}\right) \operatorname{Re} \iint_{\Omega}\left(\frac{\varkappa}{\alpha}-\bar{\mu} \varkappa\right)(\alpha-\mu) \varphi \frac{d x d y}{\left(1-|x|^{2}\right)\left(1-|\mu|^{2}\right)} \leqq \iint_{\Omega}|\alpha-\mu|^{2}|\varphi| \frac{d x d y}{\left(1-|\chi|^{2}\right)\left(1-|\mu|^{2}\right)}$.

Another version of $(\mathrm{M})$ that we shall require contains a grouping of terms for which $\varphi$ always occurs in the combination

$$
|\varphi|-\frac{x}{|x|} \varphi
$$

To achieve this we first rewrite the numerator of the integrand on the left side of $\left(\mathrm{M}^{\prime}\right)$ as

$$
\left(\frac{x}{\alpha}-\bar{\mu} x\right)(\alpha-\mu) \varphi=\frac{\bar{x}}{|x|}\left(\frac{\varkappa}{\alpha}-\bar{\mu} x\right)(\alpha-\mu) \frac{\varkappa}{|\varkappa|} \varphi=\frac{\bar{\alpha}}{|\alpha|}(1-\bar{\mu} \alpha)(\alpha-\mu) \frac{\varkappa}{|x|} \varphi .
$$

Thus, $\left(\mathrm{M}^{\prime}\right)$ can be rewritten as

where

$$
\begin{gathered}
\operatorname{Re} \iint_{\Omega} \frac{\bar{\alpha}}{|\alpha|}(1-\bar{\mu} \alpha)(\alpha-\mu)\left(|\varphi|-\frac{\varkappa}{|\varkappa|} \varphi\right) \frac{d x d y}{\left(1-|x|^{2}\right)\left(1-|\mu|^{2}\right)} \\
\geqq \iint_{\Omega} \gamma|\varphi| \frac{d x d y}{\left(1-|x|^{2}\right)\left(1-|\mu|^{2}\right)},
\end{gathered}
$$

$$
\begin{gathered}
\gamma=\operatorname{Re}\left[\frac{\bar{\alpha}}{|\alpha|}(1-\bar{\mu} \alpha)(\alpha-\mu)\right]-|\alpha-\mu|^{2}=|\alpha|\left[1+|\mu|^{2}-\left(1+\frac{1}{|\alpha|^{2}}\right) \operatorname{Re}(\bar{\mu} \alpha)\right] \\
-|\alpha-\mu|^{2}=(1-|\alpha|)\left[|\alpha|-|\mu|^{2}-(1-|\alpha|) \operatorname{Re} \frac{\bar{\mu} \alpha}{|\alpha|}\right] \\
=(1-|\alpha|)\left[|\alpha|^{2}-|\mu|^{2}+(1-|\alpha|)\left(|\alpha|-\operatorname{Re} \frac{\bar{\mu} \alpha}{|\alpha|}\right)\right] .
\end{gathered}
$$

Therefore, (M) is equivalent to the inequality

$$
\begin{aligned}
& \iint_{\Omega} \frac{\left(|\alpha|^{2}-|\mu|^{2}\right)+(1-|\chi|)\left(|\alpha|-\operatorname{Re} \frac{\bar{\mu} \alpha}{|\alpha|}\right)}{(1+|\chi|)\left(1-|\mu|^{2}\right)}|\varphi| d x d y \\
& \leqq \operatorname{Re} \iint_{\Omega} \frac{\bar{\alpha}}{|\alpha|}(1-\bar{\mu} \alpha)(\alpha-\mu)\left(|\varphi|-\frac{\varkappa}{|\chi|} \varphi\right) \frac{d x d y}{\left(1-|\chi|^{2}\right)\left(1-|\mu|^{2}\right)} .
\end{aligned}
$$

Then

Lemma 2.1. Suppose (M) is satisfied, with $|\alpha(z)| \equiv|\varkappa(z)| \equiv k$, and $|\mu(z)| \leqq k$.

$$
\iint_{\Omega}|\alpha-\mu|^{2}|\varphi| d x d y \leqq \frac{2\left(1+k^{2}\right)}{(1+k)(1-k)^{3}} \int_{\Omega} \int|\alpha-\mu| \cdot|k| \varphi|-\varkappa \varphi| d x d y .
$$


Proof. We make use of $(\mathrm{M})$ in the version $\left(\mathrm{M}^{\prime \prime}\right)$. An upper bound for the right hand side of $\left(\mathrm{M}^{\prime \prime}\right)$ is

$$
\begin{aligned}
& \frac{1}{k} \int_{\Omega}|1-\bar{\mu} \alpha| \cdot|\alpha-\mu| \cdot|k| \varphi|-\varkappa \varphi| \frac{d x d y}{\left(1-k^{2}\right)\left(1-|\mu|^{2}\right)} \\
& \quad \leqq \frac{\left(1+k^{2}\right)}{\left(1-k^{2}\right)^{2} k} \iint_{\Omega}|\alpha-\mu| \cdot|k| \varphi|-\chi \varphi| d x d y .
\end{aligned}
$$

A lower bound for the left side of $\left(\mathrm{M}^{\prime \prime}\right)$ is

$$
\frac{(1-k) k}{1+k} \iint_{\Omega}\left(1-\operatorname{Re} \frac{\bar{\mu}}{\bar{\alpha}}\right)|\varphi| d x d y .
$$

But, since $|\alpha|=k \geqq|\mu|$,

$$
\begin{aligned}
|\alpha-\mu|^{2}=|\alpha|^{2}+|\mu|^{2}-2 \operatorname{Re}(\bar{\mu} \alpha) & =k^{2}+|\mu|^{2}-2 k^{2} \operatorname{Re}\left(\frac{\bar{\mu}}{\bar{\alpha}}\right) \leqq 2 k^{2}-2 k^{2} \operatorname{Re}\left(\frac{\bar{\mu}}{\bar{\alpha}}\right) \\
= & 2 k^{2}\left(1-\operatorname{Re} \frac{\bar{\mu}}{\bar{\alpha}}\right) .
\end{aligned}
$$

If we substitute this into (2.6), and use (2.5), we obtain (2.4).

\section{Proof of Theorem A and Theorem B}

Suppose $g(z)$ is a quasiconformal mapping of $\Omega$ competing with $f(z)$, and suppose the maximal dilatation of $g$ is no greater than that of $f$. Then $\left(\mathrm{M}^{*}\right)$ holds for all $\varphi \in \mathscr{B}(\Omega)$, with $\alpha(z), \mu(z)$ as per (2.1). Since we are assuming that $f(z)$ is of Teichmüller type (1.1), we have $|\varkappa(z)| \equiv|\alpha(z)| \equiv k,|\mu(z)| \leqq k$. Therefore the hypothesis of Lemma 2.1 is satisfied, and (2.4) holds for all $\varphi \in \mathscr{B}(\Omega)$. To prove Theorem A we shall apply (2.4) to the sequence $\left\{\varphi_{n}\right\}$ specified by (1.2) and (1.3), while to prove Theorem B we make use of the sequence $\left\{\varphi_{n}\right\}$ postulated to satisfy (1.4)-(1.6). In either case our objective is to show that we must have $g=f$, or, equivalently, $g^{-1}=f^{-1}$. That is, we must show that $\hat{\varkappa}(w) \equiv \chi_{1}(w)$ a.e., or, equivalently, that

Let us set

$$
\alpha(z) \equiv \mu(z) \quad \text { a.e. in } \Omega \text {. }
$$

$$
\varrho(z)=|\alpha(z)-\mu(z)|^{2} .
$$

It will be sufficient to show that

$$
\lim _{n \rightarrow \infty} \iint_{\Omega} \varrho(z)\left|\varphi_{n}(z)\right| d x d y=0,
$$

since by Fatou's lemma, it then follows by either (1.2) or (1.4) that

and this implies (3.1).

$$
\iint_{\Omega} \varrho(z)\left|\varphi_{0}(z)\right| d x d y=0
$$


For any complex number $w$,

$$
|| w|-w|^{2}=2|w|(|w|-\operatorname{Re} w) .
$$

Therefore, (2.4), with $\varphi=\varphi_{n}$, is equivalent to

$$
\begin{gathered}
\iint_{\Omega} \varrho\left|\varphi_{n}\right| d x d y \leqq C^{1 / 2} \iint_{\Omega} \varrho^{1 / 2}\left|\varphi_{n}\right|^{1 / 2}\left[k\left|\varphi_{n}\right|-\operatorname{Re}\left(\varkappa \varphi_{n}\right)\right]^{1 / 2} d x d y, \\
C=\frac{8 k\left(1+k^{2}\right)^{2}}{(1+k)^{2}(1-k)^{6}} .
\end{gathered}
$$

Squaring, applying Schwarz's inequality, and dividing by $\iint_{\Omega} \varrho\left|\varphi_{n}\right| d x d y$, yields the estimate,

$$
\iint_{\Omega} \varrho\left|\varphi_{n}\right| d x d y \leqq C \delta\left\{\varphi_{n}\right\}
$$

Since (1.3) and (3.4) imply (3.2), Theorem A is proved.

To proceed with the proof of Theorem B, we will use (3.3) in a more refined manner while at the same time applying (3.4) as a preliminary estimate.

Let $\left\{\Omega_{m}\right\}$ be an exhaustion of $\Omega$ by an increasing sequence of compact sets. For given values of $m, n, A$, we decompose $\Omega$ into

where

$$
\Omega=\Omega^{\prime} \cup \Omega^{\prime \prime} \cup \Omega^{\prime \prime \prime},
$$

$$
\begin{aligned}
& \Omega^{\prime}=\Omega(n, A)=\left\{z \in \Omega:\left|\varphi_{n}(z)\right| \geqq A\left|\varphi_{0}(z)\right|\right\}, \\
& \Omega^{\prime \prime}=\left\{z \in \Omega \backslash \Omega_{m}:\left|\varphi_{n}(z)\right|<A\left|\varphi_{0}(z)\right|\right\}, \\
& \Omega^{\prime \prime \prime}=\left\{z \in \Omega_{m}:\left|\varphi_{n}(z)\right|<A\left|\varphi_{0}(z)\right|\right\} .
\end{aligned}
$$

Corresponding to this decomposition, we write

$$
I_{n}=\iint_{\Omega} \varrho^{1 / 2}\left|\varphi_{n}\right|^{1 / 2}\left[k\left|\varphi_{n}\right|-\operatorname{Re}\left(\varkappa \varphi_{n}\right)\right]^{1 / 2} d x d y=J_{1}+J_{2}+J_{3} .
$$

Evidently,

$$
J_{1}^{2} \leqq \int_{\Omega^{\prime}} \varrho\left|\varphi_{n}\right| d x d y \iint_{\Omega^{\prime}}\left[k\left|\varphi_{n}\right|-\operatorname{Re}\left(x \varphi_{n}\right)\right] d x d y \leqq \delta\left\{\varphi_{n}\right\} \iint_{\Omega(n, A)} \varrho\left|\varphi_{n}\right| d x d y
$$

Since $\varrho(z) \leqq(2 k)^{2}$, and in view of $(1.5)$,

$$
J_{1}^{2} \leqq 4 k^{2} M \iint_{\Omega(n, A)}\left|\varphi_{n}(z)\right| d x d y .
$$

For $J_{2}$ we have, similarly,

$$
J_{2}^{2} \leqq \delta\left\{\varphi_{n}\right\} \iint_{\Omega \backslash \Omega_{m}} \varrho\left|\varphi_{n}\right| d x d y \leqq A M \iint_{\Omega \backslash \Omega_{m}} \varrho\left|\varphi_{0}\right| d x d y .
$$

Turning our attention to $J_{3}$, we note, firstly, that, for $z \in \Omega^{\prime \prime \prime}$,

$$
k\left|\varphi_{n}(z)\right|-\operatorname{Re}\left[\varkappa(z) \varphi_{n}(z)\right]=\min \left\{k\left|\varphi_{n}(z)\right|-\operatorname{Re}\left[\varkappa(z) \varphi_{n}(z)\right], k A\left|\varphi_{0}(z)\right|\right\} .
$$


Hence, using (3.4), with Schwarz's inequality,

$$
\begin{aligned}
& J_{3}^{2} \leqq \iint_{\Omega^{\prime \prime}} \varrho\left|\varphi_{n}\right| d x d y \iint_{\Omega^{\prime \prime}}\left[k\left|\varphi_{n}\right|-\operatorname{Re}\left(\varkappa \varphi_{n}\right) \mid d x d y\right. \\
& \leqq C M \iint_{\Omega_{m}} \min \left\{k\left|\varphi_{n}\right|-\operatorname{Re}\left(\varkappa \varphi_{n}\right), k A\left|\varphi_{0}\right|\right\} d x d y .
\end{aligned}
$$

Let $\varepsilon>0$ be given.

By (1.6) and (3.6), we can choose $A$ so that

$$
J_{1}<\varepsilon / 3
$$

for all $n$. By Fatou's lemma, and (3.4),

$$
\iint_{\Omega} \varrho\left|\varphi_{0}\right| d x d y \leqq C M<\infty .
$$

Hence (3.7) shows that we can choose $m$ so that

$$
J_{2}<\varepsilon / 3
$$

for all $n$. Having chosen $A$ and $m$ we now apply Lebesgue's convergence theorem to (3.8). By the second part of hypothesis (1.4),

$$
\iint_{\Omega_{m}}\left|\varphi_{0}(z)\right| d x d y<\infty .
$$

Thus, the first part of hypothesis (1.4) implies that the right side of (3.8) has limit 0 , as $n \rightarrow \infty$. Choosing $N$ such that

we obtain, by (3.5),

$$
J_{3}<\varepsilon / 3, \quad n \geqq N,
$$

$$
I_{n}<\varepsilon, \quad n \geqq N .
$$

Hence, by (3.3), the required conclusion (3.2) follows.

\section{The affine stretch}

If $K=(1+k) /(1-k)>1$, and if $f(z)$ is the affine stretch

$$
f(z)=K x+i y, \quad z=x+i y \in \Omega,
$$

then $\varkappa(z)$ has the form (1.1), with $\varphi_{0}(z) \equiv 1$. Either Theorem A or Theorem B can be used to give sufficient conditions for the unique extremality of (4.1). The following consequence of Theorem $\mathbf{B}$ is useful in a number of situations. 
Theorem 4.1. Suppose there exists a uniformly bounded sequence of functions $\left\{\varphi_{n}\right\}, \varphi_{n} \in \mathscr{B}(\Omega)$, such that

and such that

$$
\lim _{n \rightarrow \infty} \varphi_{n}(z)=1 \quad \text { a.e. } \quad \Omega,
$$

$$
\operatorname{Re} \iint_{\Omega}\left[\left|\varphi_{n}(z)\right|-\varphi_{n}(z)\right] d x d y<M<\infty, \quad n=1,2, \ldots
$$

Then the affine stretch (4.1) of $\Omega$ is uniquely extremal.

As a special case, we obtain, for instance,

Theorem 4.2. Suppose $\Omega$ is a region with the properties,

$$
\iint_{\Omega} e^{-t y} d x d y<\infty, \text { for all } t>0 \text {, inf }\{\operatorname{Im} z: z \in \Omega\}>-\infty .
$$

If

$$
\varliminf_{t \rightarrow 0} \iint_{\Omega} e^{-t y} \sin ^{2} \frac{t x}{2} d x d y<\infty,
$$

then the affine stretch (4.1) of $\Omega$ is uniquely extremal.

Proof. Apply Theorem 4.1 with $\varphi_{n}(z)=e^{i t z}, t=t_{n} \backslash 0$, where $\left\{t_{n}\right\}$ is chosen such that

exists as a finite value.

$$
\lim _{n \rightarrow \infty} \iint_{\Omega} e^{-t_{n} y} \sin ^{2} \frac{t_{n} x}{2} d x d y
$$

Example. $\Omega_{\alpha}=\left\{(x, y): y>|x|^{\alpha}\right\}, \alpha>1$.

We will see that Theorem 4.2 is sharp.

Consider

$$
I(t)=\iint_{\Omega_{\alpha}} e^{-t y} \sin ^{2} \frac{t x}{2} d x d y=\frac{2}{\alpha t^{1+(1 / \alpha)}} \int_{0}^{\infty} e^{-\xi} \xi^{(1 / \alpha)-1} \sin ^{2}\left(\frac{t^{1-(1 / \alpha)} \xi^{1 / \alpha}}{2}\right) d \xi .
$$

It is easily verified that

$$
\lim _{t \rightarrow 0} t^{(3 / \alpha)-1} I(t)=\frac{1}{2 \alpha} \int_{0}^{\infty} \xi^{(3 / \alpha)-1} e^{-\xi} d \xi .
$$

Thus,

$$
\lim _{t \rightarrow 0} I(t)=\left\{\begin{array}{l}
\infty, \quad 1<\alpha<3, \\
1 / 6, \quad \alpha=3, \\
0, \quad \alpha<3 .
\end{array}\right.
$$

On the basis of Theorem 4.2 we conclude that the affine stretch of $\Omega_{\alpha}$ is uniquely extremal if $\alpha \geqq 3$. In fact, it is known [1] that the affine stretch of $\Omega_{\alpha}$ is uniquely extremal if and only if $\alpha \geqq 3$. 


\section{The affine stretch of a punctured plane}

In [4] Strebel and the author consider the question of the extremality of the affine stretch (4.1) for regions $\Omega=C \backslash S$ consisting of the complex plane $C$ from which a set $S$ of countably many isolated "punctures" has been removed. ${ }^{4}$ Loosely speaking, if $S$ is sufficiently dense at $\infty$, while at the same time tending to surround $\infty$ in a certain sense then (4.1) turns out to be extremal. Not surprisingly, a further increase in density of $S$ near $\infty$ suffices to imply unique extremality of the affine stretch. We will restrict ourselves here to exhibiting this phenomenon for a relatively symmetric example: We assume that $S$ contains $n$ equally spaced points on the circle ${ }^{5}$ $\left|z-c_{n}\right|=R_{n}, n=1,2, \ldots, \lim R_{n}=\infty$. Under this assumption it is known [4] that the affine stretch of $C \backslash S$ is extremal.

Theorem 5.1. Let $S$ be the discrete point set defined above. If ${ }^{5}$

$$
\frac{R_{n}^{2}}{n}, \quad n=1,2, \ldots
$$

is a bounded sequence, and if $\varlimsup_{n \rightarrow \infty}\left|c_{n}\right| / R_{n}<1$, then the affine stretch of $C \backslash S$ is uniquely extremal.

Before proceeding with the proof we require two lemmas.

Lemma 5.1. $\iint_{C}\left|1-z^{n}\right|^{-1} d x d y=\pi+O(1 / n), \quad n \rightarrow \infty$.

Proof. Since the integrand is invariant under a rotation of $C$ through an angle $2 \pi / n$,

$$
J_{n}=\iint_{C} \frac{d x d y}{\left|1-z^{n}\right|}=n \underset{\substack{0<\arg z<\frac{2 \pi}{n} \\\left|1-z^{n}\right|}}{\int} \frac{d x d y}{n} \iint_{C} \frac{d \zeta d \eta}{|\zeta|^{2-(2 / n)}|1-\zeta|}
$$

Let $F(r)$ denote the elliptic integral

$$
F(r)=\int_{0}^{2 \pi} \frac{d \theta}{\left|1-r e^{i \theta}\right|}, \quad r>0, \quad r \neq 1
$$

Evidently,

$$
F(r)=\frac{1}{r} F\left(\frac{1}{r}\right), \quad r \neq 0,1
$$

${ }^{4}$ If $S$ consists of only finitely many points then the affine stretch cannot be extremal.

${ }^{5}$ For the criteria for extremality and unique extremality subsequences suffice. Thus $n$ need only run over an infinite sequence of positive integers. 
By (5.1), (5.3),

$$
\begin{gathered}
J_{n}=\frac{1}{n} \int_{0}^{\infty} r^{(2 / n)-1} F(r) d r=\frac{1}{n} \int_{0}^{1}\left(r^{(2 / n)-1}+r^{-2 / n}\right) F(r) d r \\
=\frac{2 \pi}{n} \int_{0}^{1} r^{(2 / n)-1} d r+\frac{1}{n}\left[\int_{0}^{1} r^{2 / n} \frac{F(r)-2 \pi}{r} d r+\int_{0}^{1} r^{-2 / n} F(r) d r\right]= \\
=\pi+\frac{1}{n} \int_{0}^{1} r^{2 / n} \frac{(r+1) F(r)-2 \pi}{r} d r+\frac{1}{n} \int_{0}^{1}\left(r^{-2 / n}-r^{2 / n}\right) F(r) d r, \quad \\
\quad n=3,4, \ldots .
\end{gathered}
$$

By (5.2),

Hence,

$$
\frac{2 \pi}{1+r} \leqq F(r) \leqq \frac{2 \pi}{1-r}, \quad 0 \leqq r<1
$$

$$
0 \leqq \frac{(r+1) F(r)-2 \pi}{r} \leqq \frac{4 \pi}{1-r}, \quad 0 \leqq r<1 .
$$

On the other hand, referring, say, to the second expression for $J_{n}$ in (5.4) we see that

$$
\int_{0}^{1} r^{-2 / 3} F(r) d r<\infty .
$$

In view of (5.5), and (5.6),

$$
0<a=\int_{0}^{1} \frac{(r+1) F(r)-2 \pi}{r} d r<\infty .
$$

Taking note of (5.6) and (5.7), we conclude from the last expression for $J_{n}$ in (5.4) that

where $a$ is given by (5.7).

$$
J_{n}=\pi+\frac{a}{n}+o\left(\frac{1}{n}\right), \quad n \rightarrow \infty,
$$

Lem ma 5.2. $\iint_{C}\left(1-z^{n}\right)^{-1} d x d y=\pi, n=3,4, \ldots$

Proof. The integral converges absolutely.

$$
\begin{aligned}
& \iint_{|z|<1} \frac{d x d y}{1-z^{n}}=\iint_{|z|<1}\left(1+z^{n}+z^{2 n}+\ldots\right) d x d y=\pi, \\
& \iint_{|z|>1} \frac{d x d y}{1-z^{n}}=-\iint_{|z|>1}\left(z^{-n}+z^{-2 n}+\ldots\right) d x d y=0 .
\end{aligned}
$$

Proof of Theorem 5.1

Let

$$
\varphi_{n}(z)=\frac{R_{n}^{n}}{R_{n}^{n}-\left(z-c_{n}\right)^{n}}, \quad n=3,4, \ldots,(z \in \Omega=C \backslash S)
$$


where we have assumed, without loss of generality, that the points of $S$ on $\left|z-c_{n}\right|=R_{n}$ are located at the roots of $\left(z-c_{n}\right)^{n}=R_{n}^{n}$. $\varphi_{n}$ belongs to $\mathscr{B}(\Omega)$, since it has first-order poles on $S$ and a pole of at least order 3 at $z=\infty$.

We shall use Theorem B, with $\varphi_{0}(z) \equiv 1$. Condition (1.4) is clearly satisfied.

We have, using Lemmas 5.1 and 5.2,

$$
\iint_{\Omega}\left|\varphi_{n}(z)\right| d x d y-\iint_{\Omega} \varphi_{n}(z) d x d y=R_{n}^{2} \iint_{C} \frac{d \xi d \eta}{\left|1-\zeta^{n}\right|}-R_{n}^{2} \iint_{C} \frac{d \xi d \eta}{1-\zeta^{n}}=R_{n}^{2} O\left(\frac{1}{n}\right)
$$

Therefore, (1.5) holds.

In order to verify (1.6), assume, say

$$
\frac{R_{n}^{2}}{n} \leqq C, \quad n=1,2, \ldots
$$

Given $\varepsilon>0$, choose $A=A(\varepsilon) \geqq 2$ and sufficiently large so that

$$
\frac{9}{4} \iint_{|w-1|<\frac{1}{A}} \frac{d u d v}{|w|^{2}|1-w|}<\frac{\varepsilon}{C}, \quad n=1,2, \ldots
$$

Since $|w-1|<1 / A$ implies $|w|^{2 / n} \leqq(3 / 2)^{2}, n=1,2, \ldots$, we have

$$
\iint_{|w-1|<\frac{1}{A}} \frac{d u d v}{|w|^{2-2 / n}|1-w|}<\frac{\varepsilon}{C}, \quad n=1,2, \ldots
$$

Let

$$
E=\left\{\zeta \in C:\left|\zeta^{n}-1\right|<\frac{1}{A}\right\},
$$

and let $E_{1}$ be the component of $E$ containing the point $\zeta=1$. Since $A>1, E_{1}$ does not intersect the rays $\arg \zeta= \pm \pi / n$. Hence $E_{1}$ is the homeomorphic image of the disk $|w-1|<1 / A$ under the conformal mapping defined by the principal branch of $\zeta=w^{1 / n}, \operatorname{Re} w>0$. Transforming the integral accordingly, we have, by (5.9),

$$
n^{2} \iint_{E_{1}} \frac{d \xi}{|1-\zeta n|}<\frac{\varepsilon}{C}, \quad n=1,2, \ldots
$$

Now, $E$ is the union of $n$ (disjoint) replicas of $E_{1}$ produced by rotating the plane through multiples of the angle $2 \pi / n$. Since the integrand of $(5.10)$ is invariant under these rotations,

$$
\iint_{E} \frac{d \xi d \eta}{\left|1-\zeta^{n}\right|}=n \iint_{E_{\mathbf{1}}} \frac{d \xi d \eta}{\left|1-\zeta^{n}\right|}<\frac{\varepsilon}{n C}
$$

Accordingly, with $\Omega(n, A)$ as defined by (1.6), and $A(\varepsilon)$ defined by $(5.8)$

$$
\iint_{\Omega(n, A)}\left|\varphi_{n}(z)\right| d x d y=R_{n}^{2} \iint_{E} \frac{d \xi d \eta}{\left|1-\zeta^{n}\right|}<\frac{\varepsilon}{C} \frac{R_{n}^{2}}{n} \leqq \varepsilon .
$$




\section{Hahn-Banach extensions of $\Lambda_{\text {}}$}

Given $x(z), z \in \Omega$, of the type (1.1), we shall say that $\mathscr{F}_{x}$ is an (M)-uniqueness family for $x$ if $\mathscr{F}_{x} \subset \mathscr{B}(\Omega)$, and if, in the nomenclature of Sections 1, 2, the following implication holds:

(6.1) (M) holds for all $\varphi \in \mathscr{F}_{\varkappa}, \quad|\alpha(z)| \equiv|\chi(z)| \equiv k, \quad|\mu(z)| \leqq k \Rightarrow \alpha(z)$

$$
\equiv \mu(z) \text { a.e. in } \Omega \text {. }
$$

Evidently, if $\mathscr{F}_{x}$ is an (M)-uniqueness family for $x$ then a quasiconformal mapping $f$ with complex dilatation $\varkappa$ is uniquely extremal. In view of the fact that the proofs of Theorems A and B involved verification of the implication (6.1), one example of an (M)-uniqueness family $\mathscr{F}_{x}$ is the family $\left\{\varphi_{n}\right\}$, where $\varphi_{n}$ satisfy (1.2) and (1.3). Another example is the family $\left\{\varphi_{n}\right\}$ satisfying (1.4)-(1.6).

Theorem C. Suppose there exists an (M)-uniqueness family for $x$. Then the Hahn-Banach extension of $\Lambda_{x}$ from $\mathscr{B}(\Omega)$ to $\mathscr{L}^{1}(\Omega)$ is unique.

Proof. Suppose $\Lambda_{\varkappa}$ has the Hahn-Banach extension

where $\|v\|_{\infty} \leqq k$. Then

$$
\iint_{\Omega} v(z) \varphi(z) d x d y, \quad \varphi \in \mathscr{L}^{1}(\Omega)
$$

Let $^{6}$

$$
\iint_{\Omega} x \varphi d x d y=\iint_{\Omega} v \varphi d x d y, \text { for all } \varphi \in \mathscr{B}(\Omega)
$$

$$
\alpha(z)=\varkappa(z), \quad \mu(z)=v(z), \quad(z \in \Omega) .
$$

We claim that with the given function $x(z)$, and the above choices of $\alpha(z)$ and $\mu(z)$, (M) holds for all $\varphi \in \mathscr{B}(\Omega)$. To prove this we use (M) in the version ( $\left.\mathrm{M}^{\prime}\right)$. We need to verify that

$$
\operatorname{Re} \iint_{\Omega}(1-\bar{v} x)(\varkappa-v) \varphi \frac{d x d y}{1-|v|^{2}} \leqq \iint_{\Omega}|x-v|^{2}|\varphi| \frac{d x d y}{1-|v|^{2}}, \quad \varphi \in \mathscr{B}(\Omega) .
$$

By (6.2),

$$
\begin{gathered}
\iint_{\Omega} \frac{(1-\bar{v} x)(x-v)}{1-|v|^{2}} \varphi d x d y=\iint_{\Omega}\left[\frac{(1-\bar{v} x)(\varkappa-v)}{1-|v|^{2}}-(\varkappa-v)\right] \varphi d x d y \\
=-\iint_{\Omega} \frac{(\varkappa-v)^{2} \bar{v}}{1-|v|^{2}} \varphi d x d y .
\end{gathered}
$$

\footnotetext{
${ }^{6}$ Alternatively, the somewhat more intuitive choice, $\alpha=-\varkappa, \mu=-v$ could be used.
} 
Therefore,

$$
\begin{gathered}
\operatorname{Re} \iint_{\Omega} \frac{(1-\bar{v} x)(\varkappa-v)}{1-|v|^{2}} \varphi d x d y \leqq \iint_{\Omega} \frac{|x-v|^{2}|v|}{1-|v|^{2}}|\varphi| d x d y \\
\leqq k \iint_{\Omega} \frac{|x-v|^{2}}{1-|v|^{2}}|\varphi| d x d y
\end{gathered}
$$

and (6.4) follows. In view of the postulated existence of an (M)-uniqueness family $\mathscr{F}_{x}$ it follows that $\alpha(z) \equiv \mu(z)$ a.e., i.e., $v(z) \equiv \varkappa(z)$ a.e., as was to be shown.

As corollaries one can use the hypotheses of any of the Theorems 4.1, 4.2, and 5.1, to conclude that the corresponding functions $\Lambda_{\varkappa}$ have unique Hahn-Banach extensions from $\mathscr{B}(\Omega)$ to $\mathscr{L}^{1}(\Omega)$. So, for example, as an analog to Theorem 4.1 one can state the following generalization of a result obtained in [3].

Theorem 4.1'. Suppose there exists a uniformly bounded sequence of functions $\left\{\varphi_{n}\right\}, \varphi_{n} \in \mathscr{B}(\Omega)$, such that (4.2) and (4.3) hold. Then the linear functional

$$
\iint_{\Omega} \varphi(z) d x d y, \quad \varphi \in \mathscr{B}(\Omega)
$$

has a unique Hahn-Banach extension from $\mathscr{B}(\Omega)$ to $\mathscr{L}^{1}(\Omega)$. Moreover, the formula (6.5) provides this extension to $\varphi \in \mathscr{L}^{1}(\Omega)$.

\section{References}

[1] Reich, E., and K. Strebel: On the extremality of certain Teichmüller mappings. - Comment Math. Helv. 45, 1970, 353-362.

[2] Reich, E., and K. Strebel: Extremal quasiconformal mappings with given boundary values. Contributions to Analysis. A collection of papers dedicated to Lipman Bers. Academic Press, New York-London, 1974, 375-391.

[3] REICH, E.: Uniqueness of Hahn-Banach extensions from certain spaces of analytic functions. Math. Z. 167, 1979, 81-89.

[4] Reich, E., and K. Strebel: Quasiconformal mappings of the punctured plane. - Notes. Forschungsinstitut für Mathematik ETH Zürich, November 1979, 31 pp.

[5] ReIch, E.: A criterion for unique extremality of Teichmüller mappings. - Indiana Univ. Math. J. $30,1981,441-447$.

[6] Strebel, K.: On quasiconformal mappings of open Riemann surfaces. - Comment. Math. Helv. 53, 1978, 301-321.

School of Mathematics

University of Minnesota

Minneapolis, Minnesota 55455

USA

Received 5 May 1981 